\title{
Correlation of serum IL-4 levels in new and MDR tuberculosis patients
}

\author{
Nazish Fatima ${ }^{1,}$ ", Mohammad Shameem ${ }^{2}$, Nabeela ${ }^{1}$, Parvez A. Khan ${ }^{1}$, H. M. Khan ${ }^{1}$ \\ ${ }^{1}$ Department of Microbiology, Jawaharlal Nehru Medical College, Aligarh Muslim University, Aligarh, India \\ ${ }^{2}$ Department of TB \& Respiratory Disease, Jawaharlal Nehru Medical College, Aligarh Muslim University, Aligarh, India
}

\section{Email address:}

nazsham28@gmail.com (N. Fatima),dr.shameem123@gmail.com (M. Shameem), k.nabeela092@gmail.com (Nabeela), parvezkhananvar@gmail.com (P. A. Khan), harismk2003@hotmail.com (H. M. Khan)

\section{To cite this article:}

Nazish Fatima, Mohammad Shameem, Nabeela, Parvez A. Khan, H. M. Khan. Correlation of Serum IL-4 Levels in New and MDR Tuberculosis Patients. American Journal of Clinical and Experimental Medicine. Vol. 3, No. 1, 2015, pp. 48-51.

doi: 10.11648/j.ajcem.20150301.17

\begin{abstract}
Mycobacterium tuberculosis (Mtb) infects approximately one third of world population. Many cytokines are produced during tuberculosis, with predominance of Th1 cytokines during the early stage and Th2 cytokines in the later stages of the infection. Most studies on cytokines during TB are from 'in vitro'-stimulated lymphoid cells with few reports on in vivo plasma levels. The high expression of IL-4 has been implicated as a virulence factor. In the present study we examined the level of IL-4 in the serum of active tuberculosis in new cases, during anti-tubercular therapy and MDR cases. This Study was conducted at Department Of Microbiology, JNMC, AMU, Aligarh. A total 76 blood samples of Pulmonary \& Extra-Pulmonary tuberculosis patients were tested by ELISA (Diaclone France) along with 10 healthy controls. The test was carried out according to the manufacturer instructions. O.D. was taken at $450 \mathrm{~nm}$. There was no stastical difference in age and sex in our study. The level of IL-4 show significant changes in MDR TB cases $(P<0.05)$ but not significant during the anti-tubercular therapy (ATT) and new TB cases $(P>0.05)$. An understanding of the development of the cellular immune response may lead to insight into pathogenesis and novel therapies for TB. Further studies are needed to address the role of cytokines in immunity to TB under natural conditions.
\end{abstract}

Keywords: MDR TB, IL-4, Cytokines

\section{Introduction}

Tuberculosis $(\mathrm{TB})$ is the major health problem throughout the world causing large number of deaths, more than that from any single infectious disease. Approximately 8-10 million people are infected with this pathogen every year [1]. In India, there are about 500,000 deaths occurring annually due to TB [2].

Tuberculosis begins with the inhalation of Mtb containing aerosols into the pulmonary alveoli. Here, the bacteria bind to phagocytic receptors and enter resident alveolar macrophages, dendritic cells, and monocytes recruited from the blood stream.

Cytokines are primarily involved in host responses to disease or infection and any involvement with homeostatic mechanism has been less than dramatic. Many cytokines are produced during TB $[3,4]$, with a predominance of Th1 cytokines during the early stage $[5,6]$. Multidrug-resistant tuberculosis (MDR-TB) has become a lethal global threat. Insights into the immune regulation of MDR-TB are urgently needed for the development of new treatments; however, the $\mathrm{T}$ cell response to an MDR-TB infection in human remains unclear [7].

Some cytokines promote inflammation and are called pro-inflammatory cytokines [TNF-alpha, IL-6, IL-8], whereas some cytokines suppress the activity of pro-inflammatory cytokines and are called anti-inflammatory cytokines.

The current testing methodologies for the diagnosis of TB are extremely slow, it can take up to 8 weeks for the accurate Mtb diagnosis to be made. As a result, there is an increasing need for both novel therapies and rapid diagnostic methods.

Diagnosis of multi-drug resistant TB is difficult especially in Extra- Pulmonary TB cases. Sample collection is difficult in Extra-Pulmonary TB cases and culture positivity rate is very low. We have to find answers for early detection of MDR-TB cases especially Extra- Pulmonary TB. Thus more 
focus is being given to serum biomarker for early detection. With this background, we undertook a study to examine the level of IL-4 in the serum of active tuberculosis in new cases, during anti-tubercular therapy and MDR Pulmonary \& ExtraPulmonary TB cases.

\section{Material Methods}

This prospective study was conducted at the Department of Microbiology, JNMC, AMU, Aligarh. Samples were collected from the out-patients and in-patients of department of TB \& Respiratory diseases, Jawaharlal Nehru Medical College, AMU, Aligarh. A complete clinical and radiological data was collected. Informed consent was taken from all Subjects. The study was approved by Institutional Bioethical Committee.

\subsection{Selection of Patients}

All sputum positive patients (new case, relapse, defaulter, treatment failure) including Tubercular effusion, pericardial effusion was enrolled.

- New case: a patient who has never taken anti-TB drugs for more than 1 month.

- Relapse: previously received treatment, cured, and has once again (+) PTB.

- Failure: a TB patient who while on treatment remains $(+)$, or becomes $(+)$ at 5 th month or later, while on treatment, or was (-) at start and becomes $(+)$ after 2 nd month of treatment.

- Treatment after Default: A patient who returns to treatment following interruption of treatment for two months or more and is positive bacteriologically.

\subsection{Sputum Culture and Drug Susceptibility Test}

Sputum culture positive for Mycobacterium tuberculosis was confirmed by inoculation of samples on Lowenstein Jensen (LJ) media. Briefly, sputum specimen were decontaminated with $4 \% \mathrm{NaOH}$ and inoculated into $\mathrm{LJ}$ media. When the growth was detected as positive, drug susceptibility test was carried out as per the absolute concentration method as previously described by G. Canetti et al (8). The reference strain H37Rv was used as control.

\subsection{Blood Collection}

76 Blood samples $(3-5 \mathrm{ml})$ were collected from department of TB \& Chest Jawaharlal Nehru Medical College, Aligarh. Samples were collected in plain vaccutainers from patients before starting anti-tuberculosis treatment (ATT) and after 2, $4 \& 8$ months of ATT. Blood samples were centrifuged at $5000 \mathrm{rpm}$ for 10 minutes and then serum were collected and stored at $-20^{\circ} \mathrm{C}$ until they were assayed. Extra-pulmonary sites were included pleural, lymph nodes, soft tissues, meninges, gastrointestinal, bone and joints and disseminated disease. Sera was also obtained from patients with TB who had been treated for at least 2 weeks, but had not yet completed therapy at the time of blood sampling and from patients who had completed anti-tuberculous therapy . Records of all patients with active TB was reviewed for clinical data as fever (rectal temperature $>38^{\circ} \mathrm{C}$ ), anorexia, skin test, bacille Calmette-Guérin (BCG) vaccination, direct microscopy, and culture results.

Furthermore, 10 sera samples were obtained from persons who had been in close contact with patients with smear-positive TB and from healthy (all skin test-negative) controls.

\subsection{Cytokines Assay}

For cytokine analysis, sandwich ELISA with monoclonal antibody sets (Diaclone SAS, Besancon Cedax, France), Streptavidin- Horse reddish peroxides conjugate and recombinant cytokines as standard were used.

Briefly, 96 well plates were coated with IL-4 according to the manufacturer's protocols. Samples were added to all the wells. Diluted biotinylated anti-IL-4 were added and incubated at room temperature for $3 \mathrm{hrs}$. After washing two times added streptavidin-HRP and incubated for 30 minutes. TMB substrate was added to each well and incubated at room temperature for 12-15 minutes and the reaction was stopped by adding stop solution. Plate was read at $450 \mathrm{~nm}$ in an ELISA reader (Thermo Electron Corporation). The detection range of the assay was less than $0.7 \mathrm{pg} / \mathrm{ml}$ for IL-4.

\subsection{Stastical Analysis}

All statistical analyses were performed using SPSS Statistics (version-20). Receiver operating characteristic (ROC) curve and other performance measures were performed using the statistical software Med Calc (version 10.2.0.0). Chi square test was done to get the significance of $P$-value. $P$-value $<0.05$ considered as significant.

\section{Results}

Of the 76 patients were enrolled, $33(43.42 \%)$ were new, $24(31.57 \%)$ were UT, 19 (25\%) were MDR TB cases, 1 (1.31\%) had pneumothorax, 2 (2.63\%) had pyopneumothorax while $1(1.31 \%)$ had diabetes mellitus. None of the patients was HIV positive. 10 BCG vaccinated healthy individuals were included as controls.

The level of IL-4 showed no significant changes in new cases $(p>0.05)$ and under-treatment cases $(p>0.05)$ as compared to controls. However, significant increase in MDR TB cases $(\mathrm{p}<0.05)$ as compared to controls was observed by Chi square test.

There were no significant variations according to the site of the involvement in Pulmonary or Extra-pulmonary TB cases (Table-1).

Table 1. Distribution of study subject in relation to site of TB

\begin{tabular}{llll}
\hline & EPTB $(\mathbf{n}=35)$ & PTB $(\mathbf{n}=\mathbf{4 1})$ & P value \\
\hline NEW & $19(54.2 \%)$ & $14(34.1 \%)$ & $P=0.104(\mathrm{NS})$ \\
Under-treatment & $13(37.1 \%)$ & $11(26.8 \%)$ & $P=0.458$ (NS) \\
MDR & $9(25.7 \%)$ & $10(24.3 \%)$ & $P=1.000$ (NS) \\
\hline
\end{tabular}




\section{Discussion}

Several studies have demonstrated the importance of cytokines as marker of TB activity. Cytokines are molecules that mediate mainly the intercellular communication in the immune system, being produced by different cell types. Cytokines have pleiotropic and regulatory effects and participate in the host defense and in inflammatory and tissue reparation processes [9]

Upon stimulation by a pathogen, macrophages engulf the offending particle, and upon its destruction, they present smaller peptide antigens on their surface. These antigens are then recognized by Th1 cells, which in turn secrete various cytokines, including IFN- $\gamma$, IL-12 and TNF- $\alpha$. These cytokines in turn activate resting macrophages, which trigger the immune response. In order to suppress the immune response once an infection has been cleared, and to prevent autoimmune responses to self-antigens, other cytokines down regulate the immune system. IL-10 activates B and Th2 cells while inhibiting Th1 cytokine production. Activated Th2 cells secrete IL-4, which also inhibits Th1 cell activity and inactivates macrophages [10].

IL-4 is produced by T-helper cells and act as a Co-factor in activation of humoral immunity by activation of B- cells and T-cells proliferation and differentiation [11, 12]. The high expression of IL-4 has been implicated as a virulence factor, both for the anti-inflammatory ability and also for its apparent capacity to promote tissue damage in association with TNF- $\alpha$ [13].

Some previous studies have shown increased production of IL-4 in human TB patients especially those with cavitary disease $[14,15]$. Whereas, some studies found no significant difference in IL-4 levels between TB patients and controls $[16,17,18,19]$. Similar to these studies we found no significant difference in IL-4 levels among new \& undertreatment TB cases compared to healthy controls.

A S Shahemabadi et al found elevated levels of IL-4 in TB patients [20]. The stimulated $\mathrm{CD}^{+} \mathrm{T}$ cells by $M$. tuberculosis may be shifted to $\mathrm{T}$ helper 2 responses in MDR-TB patients. Thus our "in vivo" study confirmed their "in vitro" findings. An "in vivo" study can better represent the interplay of various cytokines within body than "ex-vivo" studies. Tan Q et al [7] also found up-regulation of IL-4 expression in their study on cytokines response in blood. We thus believe, IL-4 can serve as a potential biomarker for early detection of MDR TB cases. Interestingly, we found significantly increased levels of IL-4 in MDR TB patients.

Several of the immune theories focus on the central role of Th1/Th2 cross regulation. In particular it has been hypothesized that there is a switch from a Th1 to a Th2-dominant cell mediated immune response leading to activate disease, as is seen in other infections. IL-4 represented as one of the cytokines which produced by $\mathrm{Th}_{2}$ cells and act as a co-factor in activation of humoral immunity by activation of B-cells and T-cells proliferation and differentiation [21]

M. tuberculosis evolved and has developed mechanisms which interact and modulate the host immune response. Mycobacterium expresses surface antigens that can induce the production of IL-4, which typically have anti-inflammatory effects $[22,23]$. The high expression of IL-4 has been implicated as a virulence factor, both for the anti-inflammatory ability and also for its apparent capacity to promote tissue damage in association with TNF- $\alpha$ [24].

These studies suggest that IL-4 may play a role in tissue destruction during infection by M. tuberculosis.

\section{Conclusions}

Serum level of Cytokines in TB patients is useful in the evaluation of the disease activity of TB during therapy.

Types of cellular immune response may affect presentation and outcome in pulmonary TB. IL-4 can use as a marker for early detection of a TB patient progressing the MDR TB.

This concept, if validated could lead to the development of clinical intervention and accelerate the conduction of clinical trials.

Further studies are required with larger cohorts to explain the mechanism of outcomes and cytokines in TB.

\section{Acknowledgement}

We are thankful to UP-Council of Science \& Technology, Lucknow, India for providing the financial assistance to carry out this research work. We are grateful to Mr. Sanjay Sharma for excellent technical expertise.

\section{References}

[1] World Health Organization. The World Health Report: Making a difference; (1999) 110.

[2] Revised National Tuberculosis Control Programme: Key facts and concepts. New Delhi: Central TB Division. Directorate General of Health Services, Ministry of Health and Family Welfare. (1999).

[3] Orme IA et al. Cytokine secretion of CD4 T lymphocytes acquired in response to Mycobacterium tuberculosis infection. J Immunol. 1993; 151: 518 /525.

[4] Kaplan G et al. The role of cytokines in the immune response to tuberculosis. Res Immunol. 1996; 147: 565-572.

[5] Dlugovitzky D et al. Circulating profile of Th1 and Th2 cytokines in tuberculosis patients with different degrees of pulmonary involvement. FEMS Immunol. 1997; 18:203-207.

[6] Orme IM. The kinetics of emergence and loss of mediator T lymphocytes acquired in response to infection with Mycobacterium tuberculosis. J Immunol. 1987; 138: 293 -298.

[7] Tan Q et al. Characterization of Th1- and Th2-type immune response in human multidrug-resistant tuberculosis. Eur J Clin Microbiol Infect Dis. 2012; 31(6):1233-42.

[8] G. Canetti et al. Mycobacteria: Laboratory methods for testing drug sensitivity \& resistance. Bul.org mond. 1963; 29: 565-578. 
[9] A. Gupta et al. Mycobacterium tuberculosis: immune evasion, latency and reactivation. Immuno-biology. 2012; 217(3): 363-374.

[10] Seok-Yong Eum et al. Association of Antigen stimulated release of TNF- $\alpha$ in whole blood in response to chemotherapy in patients with Active Pulmonary MDR tuberculosis. Respiration. 2010; 80(4): 275-284.

[11] Mossman \& Sad. 1998.

[12] Surcel HM et al. Th1/Th2 profiles in tuberculosis, based on the proliferation and cytokine response of blood lymphocytes to mycobacterial antigens. Immunology. 1994; 81(2):171-176.

[13] G. T. Seah et al. IL-4 influences apoptosis of mycobacterium-reactive lymphocytes in the presence of TNF- $\alpha$. Journal of Immunology. 200; 167 (3):1230-1237.

[14] Lin $\mathrm{Y}$ et al. Absence of a prominent Th2 cytokine response in human tuberculosis. Infect Immun. 1996; 64(4):1351-1356.

[15] Zhang M, Lin Y, Iyer DV, Gong J, Abrams JS, Barnes PF. T-cell cytokine responses in human infection with mycobacterium tuberculosis. Infect Immun. 1995; 63(8):3231-3234.

[16] Verbon A et al. Serum concentrations of cytokines in patients with active tuberculosis (TB) and after treatment. Clin Exp Immunol. 1999; 115(1):110-113.
[17] Condos $\mathrm{R}$ et al. Local immune responses correlate with presentation and outcome in tuberculosis. Am J Respir Crit Care Med. 1998; 157:729-735.

[18] Zhong $\mathrm{D}$ et al. Alteration of interferon-gamma and interleukin-12 released by bronchoalveolar lavage cells from pulmonary tuberculosis. Zhonghua Jie $\mathrm{He} \mathrm{He} \mathrm{Hu} \mathrm{Xi} Z \mathrm{Za} Z$ Zhi. 2000; 23(9):552-555.

[19] C. Manca et al. Differential monocyte activation underlies strain-specific Mycobacterium tuberculosis pathogenesis. Infection and Immunity. 2004; 72 (9):5511-5514.

[20] A S Shahemabadi et al, Evaluation of $\mathrm{T}$ cell immune responses in multi-drug resistant tuberculosis patients to mycobacterium tuberculosis total lipid antigen, Clin Exp Immunol. August 2007; 149(2):285-294.

[21] Lin $\mathrm{Y}$ et al. Absence of a prominent Th2 cytokine response in human tuberculosis. Infect Immun. 1996; 64(4):1351-1356.

[22] Y. van Kooyk and T. B. H. Geijtenbeek. DC-SIGN: escape mechanism for pathogens. Nature Reviews Immunology. 2003; 3 (9):697-709.

[23] G. T. Seah and G. A. W. Rook. IL-4 influences apoptosis of mycobacterium-reactive lymphocytes in the presence of TNF- $\alpha$. Journal of Immunology.2001; 167 (3)1230-1237.

[24] S. Stenger. Immunological control of tuberculosis: role of tumour necrosis factor and more. Annals of the Rheumatic Diseases.2005; 64 (4) 24-28. 